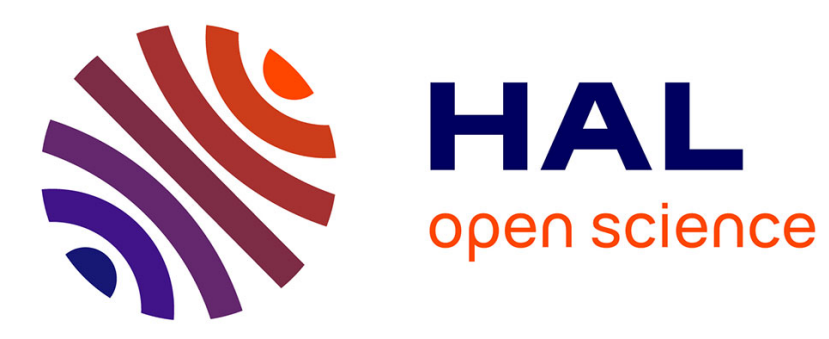

\title{
On the ultrasonic absorption and dispersion in amorphous superconductors
}

\author{
W. Arnold, P. Doussineau, Ch. Frénois, A. Levelut
}

\section{To cite this version:}

W. Arnold, P. Doussineau, Ch. Frénois, A. Levelut. On the ultrasonic absorption and dispersion in amorphous superconductors. Journal de Physique Lettres, 1981, 42 (13), pp.289-294. 10.1051/jphyslet:019810042013028900 . jpa-00231930

\section{HAL Id: jpa-00231930 https://hal.science/jpa-00231930}

Submitted on 1 Jan 1981

HAL is a multi-disciplinary open access archive for the deposit and dissemination of scientific research documents, whether they are published or not. The documents may come from teaching and research institutions in France or abroad, or from public or private research centers.
L'archive ouverte pluridisciplinaire HAL, est destinée au dépôt et à la diffusion de documents scientifiques de niveau recherche, publiés ou non, émanant des établissements d'enseignement et de recherche français ou étrangers, des laboratoires publics ou privés. 


\title{
On the ultrasonic absorption and dispersion in amorphous superconductors
}

\author{
W. Arnold (*), P. Doussineau (**), Ch. Frénois $\left({ }^{* *}\right)$ and A. Levelut (**) \\ (*) Fraunhofer Institute for Non Destructive Testing, Bldg 37, University, 6600 Saarbruecken, Federal Republic of Germany \\ (**) Laboratoire d'Ultrasons (***), Université Pierre-et-Marie-Curie, Tour 13, 4, place Jussieu, 75230 Paris Cedex 05, France
}

(Reçu le 27 février 1981, accepté le 11 mai 1981)

\begin{abstract}
Résumé. - Nous expliquons le comportement inattendu de l'absorption ultrasonore observé dans le supraconducteur amorphe $\mathrm{Pd}_{30} \mathrm{Zr}_{70}$. Nous supposons que la relaxation des états tunnel, présents dans les amorphes métalliques, est déterminée par leur fort couplage avec les électrons aussi bien qu'avec les phonons thermiques. La distribution des constantes de couplage correspondantes telle que la prévoit le modèle des états tunnel conduit à une variation faible de l'absorption avec la température lorsque la température de transition supraconductrice $T_{\mathrm{c}}$ est franchie. A l'opposé, la variation de vitesse devrait montrer un changement à $T_{\mathrm{c}}$.

Abstract. - We explain the unexpected behaviour of the ultrasonic absorption observed in the amorphous superconductor $\mathrm{Pd}_{30} \mathrm{Zr}_{70}$. It is assumed that the relaxation of the tunnelling states present in amorphous metals, is determined by their strong coupling to electrons as well as to thermal phonons. The distribution of the corresponding coupling strengths as invoked in the tunnelling model leads to a smooth temperature dependence of the absorption when passing through the superconducting transition temperature $T_{\mathrm{c}}$. In contrast, the dispersion should exhibit a change of slope at $T_{c}$.
\end{abstract}

1. Introduction. - The existence of low-energy excitations which can be regarded as two level systems (T.L.S.) is well established in amorphous metals [1] as well as in amorphous dielectrics. In the amorphous network certain atoms or groups of atoms can reside in double wells and are at the origin of these T.L.S. At low temperatures the transition between the energy levels occurs via tunnelling. As in amorphous insulators, metallic glasses exhibit in the very low temperature range $(T<1 \mathrm{~K})$ the typical acoustic behaviour :

1) a logarithmic temperature dependence of the relative variation of the sound velocity [2];

2) saturation of the resonant part of the sound attenuation when the acoustic flux exceeds a certain critical value. This critical value is, however, several orders of magnitude larger than in insulating glasses indicating that an hitherto unknown mechanism plays an important role in the relaxation process of the T.L.S. [3]. It has been suggested that the electrons are at the origin of this new mechanism [4] ;

3) relaxational attenuation due to T.L.S. which,

(***) Associated with the Centre National de la Recherche Scientifique. however, does not increase as strongly with temperature as in amorphous insulators [1].

Other experiments such as measurements of specific heat and thermal conductivity are also of great interest, but entail certain difficulties in interpretation. The T.L.S. are expected to give a linear contribution $\left(C_{\mathrm{p}} \propto T\right)$ to the specific heat and a $T^{2}$-dependence for the thermal conductivity as in amorphous insulators. Since the electrons always present in a metal give exactly the same temperature dependence for these two quantities, any T.L.S. contribution can be obscured.

An appealing possibility which avoids this difficulty is to carry out experiments in a metallic glass which is superconducting [15]. Because of the pair condensation for electrons, it is expected that their role diminishes rapidly below the transition temperature $T_{c}$.

The effect of superconductivity on the acoustic properties of metallic glasses has been calculated recently [5]. For instance the absorption of ultrasound is predicted to become very small for $T \ll T_{\text {c }}$ relative to the value in the normal state. It should increase rapidly when $T$ approaches $T_{\mathrm{c}}$. In [5] the origin of this effect is explained in the following way : the acoustic wave perturbs the population difference 
of the T.L.S. and therefore gives rise to a relaxational absorption process. In the normal state the relaxation time $T_{1}$ of the T.L.S. is extremely short because of the strong coupling between T.L.S. and electrons [4]. In the superconducting state this relaxation channel disappears : $T_{1}$ becomes longer and the resulting sound attenuation is modified greatly.

From the experimentalists' point of view an attractive possibility is offered : by varying the value of a magnetic field $H$ applied simultaneously with the ultrasound superconductivity can be switched on or off depending on whether $H$ is lower or higher than the critical field $H_{\mathrm{c}_{2}}$. Therefore for any $T<T_{\mathrm{c}}$ the normal state relaxation rate can be easily recovered provided $H>H_{\mathrm{c}_{2}}$ making it possible to gain information on the role of the electrons in the absorption process.

Such an experiment has indeed been carried out recently on a $\mathrm{PdZr}$ alloy [6] using longitudinal waves of frequencies $\omega / 2 \pi=0.74 \mathrm{GHz}$ and $1.3 \mathrm{GHz}$. The critical temperature of this compound is $T_{\mathrm{c}}=2.62 \mathrm{~K}$. As expected, at very low temperatures $(T=0.4 \mathrm{~K})$ the relaxation attenuation becomes very small, and it increases drastically after applying a magnetic field of the order of $H_{c_{2}}$. Increasing the temperature, the attenuation rises rapidly and reaches the normal state value at a temperature which, surprisingly, turns out to be not equal to $T_{\mathrm{c}}$ but much lower $(\simeq 1.5 \mathrm{~K})$ (Fig. 2). It has been checked that the critical temperature of the sample (thin platelets) is $T_{\mathrm{c}}=2.62 \mathrm{~K}$ and sharp within $\pm 0.05 \mathrm{~K}$. In this paper we present an explanation of this unexpected behaviour. We show that it is necessary to take into account not only the relaxation of the T.L.S. by thermally excited quasi-particles still present at $T<T_{\text {c }}$, but also the relaxation of the T.L.S. by the thermal phonons. Moreover, it is necessary to involve a stronger T.L.S.-electron coupling than previously assumed [5] $\left(^{1}\right)$.

2. Theory and numerical calculations. - When we consider the relaxational attenuation due to T.L.S. in amorphous metals two points must be kept in mind :

1) In metallic glasses the sound velocity of transverse waves is small as compared to amorphous insulators. As a consequence the relaxation processes due to phonons are strongly enhanced because the sound velocity $v$ enters as $v^{-5}$ in the direct process, which dominates at low temperatures.

2) Because of the distribution of the coupling strength between T.L.S. and electrons as well as

( $\left.{ }^{1}\right)$ A short version of the physical ideas and numerical calculations presented in this paper has been given by W. Arnold and G. Weiss at the International Conference on Ultrasound in Condensed Matter Physics at Zilina, CSSR (1980), to be published in Phys. Acta Slovaca. between T.L.S. and phonons, the condition $\omega T_{1} \ll 1$ cannot be fulfilled at a given temperature for all T.L.S. This consequence of the distribution function has been discussed previously [7] and worked out in detail for the superionic conductor $\beta$-alumina [8]. The important feature is that the ultrasonic attenuation becomes constant as a function of temperature provided $\omega T_{1}^{\mathrm{m}} \ll 1$ where $T_{1}^{\mathrm{m}}$ is the minimum relaxation time of the T.L.S. ( $T_{1}^{\mathrm{m}}$ originates from T.L.S. in double wells having no asymmetry). Furthermore, the attenuation does not depend on the details of the relaxation process when $\omega T_{1}^{\mathrm{m}} \ll 1$. As a consequence, even if the dominant relaxation process changes rapidly with temperature, this does not influence the attenuation as a function of temperature. The height of this plateau depends linearly on frequency.

Our explanation of the experimental results is based on the following hypotheses :

a) When the relaxation of the T.L.S. caused by the electrons is dominant $\left(H>H_{\mathbf{c}_{2}}\right)$, the relaxation rate becomes so strong that even at the lowest temperatures $(T=0.4 \mathrm{~K})$ the condition $\omega T_{1}^{\mathrm{m}} \ll 1$ holds. As mentioned above, the attenuation is then weakly temperature-dependent.

b) When the relaxation of the T.L.S. by electrons is suppressed $\left(H=0, T<T_{\mathrm{c}}\right)$, the contribution of the thermal phonons to the relaxation rate becomes noticeable. At the lowest temperatures it manifests itself by a $T^{3}$-dependence of the attenuation, indicating that as in insulators the direct (one phonon) process is dominant [7].

c) At higher temperatures $(T \gtrsim 1.5 \mathrm{~K})$ the relaxation rate even without any applied field is so strong that the condition $\omega T_{1}^{\mathrm{m}} \ll 1$ is fulfilled and a plateau is obtained for the attenuation.

We have carried out numerical calculations using the same routine and notation as in [8]. It is assumed that there is a distribution of the coupling strength described by the parameter $r=\left(\Delta_{0} / E\right)^{2}$. Here $2 E$ is the energy splitting of a given T.L.S. and $\Delta_{0} / \hbar$ is its tunnelling frequency. As a consequence all the relaxation times relevant to the problem can be written as $1 / T_{1}=r / T_{1}^{\mathrm{m}}$. The relaxation attenuation is given by the following equation :

$$
\begin{aligned}
l^{-1}=\frac{\omega}{v} & \frac{\mathcal{N} \gamma^{2}}{\rho v^{2}} \int_{k_{\mathrm{m}}}^{E_{\mathrm{M}}} \mathrm{d}(\beta E) \operatorname{sech}^{2}(\beta E) \times \\
& \times \int_{r_{\mathrm{m}}}^{1} \mathrm{~d} r \frac{(1-r) P(E, r) \omega T_{1}(E, r, T)}{1+\omega^{2} T_{1}^{2}(E, r, T)} .
\end{aligned}
$$

Here $\mathcal{N}$ is the number of T.L.S. per unit volume, $\rho$ is the density of the material, $v$ is the sound velocity, $\beta=(k T)^{-1}, \gamma$ is the deformation potential between T.L.S. and phonons, and $P(E, r)$ is the distribution function of the parameters $E$ and $r$. We used the sim- 
plest distribution plausible as deduced in the original paper of Phillips [9] :

$P(E, r)=$

$\left\{\begin{array}{ll}\frac{1}{2} A(1-r)^{-1 / 2} r^{-1} & r_{\mathrm{m}} \leqslant r \leqslant 1 \quad E_{\mathrm{m}} \leqslant E \leqslant E_{\mathrm{M}} \\ 0 & \text { elsewhere }\end{array}\right\}$

with $E_{\mathrm{m}}=\Delta_{\mathrm{m}}$ and $r_{\mathrm{m}}=\left(\Delta_{\mathrm{m}} / E\right)^{2}$ where $\Delta_{\mathrm{m}}$ is the minimum value of $\Delta_{0}$. Similarly, the relative velocity variation is given by :

$$
\begin{array}{rl}
\frac{\Delta v}{v}=-\frac{1}{2} \frac{\mathcal{N} \gamma^{2}}{\rho v^{2}} \int_{L_{\mathrm{m}}}^{L_{\mathrm{M}}} & \mathrm{d}(\beta E) \operatorname{sech}^{2}(\beta E) \times \\
& \times \int_{r_{\mathrm{m}}}^{1} \mathrm{~d} r \frac{(1-r) P(E, r)}{1+\omega^{2} T_{1}^{2}(E, r, T)} .
\end{array}
$$

In equations (1) and (3) it is the temperature dependence of the relaxation rates which determines $l^{-1}(T)$ and $\Delta v(T) / v$. These relaxation rates are :

1. ONE PHONON OR DIRECT PROCESS :

$$
\begin{gathered}
\frac{1}{T_{1}^{\mathrm{D}}}=r K_{3} T^{3} F_{3}(\beta E) \\
K_{3}=\frac{4 k^{3}}{\pi \hbar^{4}} \sum_{\tau} \frac{\gamma_{\tau}^{2}}{\rho v_{\tau}^{5}} \quad F_{3}(u)=u^{3} \operatorname{coth} u
\end{gathered}
$$

( $\tau$ designates the polarization, $\tau=\mathrm{L}$ or $\mathrm{T}$ ).

2. Relaxation of T.L.S. BY electrons IN THE NORMAL STATE (KORRINGA PROCESS) :

$$
\begin{aligned}
\frac{1}{T_{1}^{\mathrm{N}}} & =r K_{1} T F_{1}(\beta E) \\
K_{1} & =\frac{\pi k}{2 \hbar}\left(\tilde{\rho} K_{\mathrm{e}}\right)^{2} \\
F_{1}(u) & =u \operatorname{coth} u .
\end{aligned}
$$

Here, $\tilde{\rho} K_{\mathrm{e}}$ is the density of states of the electrons at the Fermi level multiplied by the deformation potential $K_{\mathrm{e}}$ between T.L.S. and electrons [1]. The linear temperature dependence of equation $(5)(E \ll k T)$ reflects the fact that only a fraction $T / T_{\mathrm{F}}$ of all electrons can contribute to $1 / T_{1}^{\mathrm{N}}\left(T_{\mathrm{F}}:\right.$ Fermi temperature).

3. RELAXATION DUE TO THERMALLY EXCITED QUASIPARTICLES $\left(T<T_{\mathrm{c}}\right)[5]$ :

$$
\frac{1}{T_{1}^{S}}=r K_{1} T F(\beta E)
$$

$$
\begin{aligned}
F(u)=\frac{1}{2} \cosh u & \{(n(u) \operatorname{sech} u) *(n(u) \operatorname{sech} u)+ \\
+ & \left.\left(\frac{\delta}{u} n(u) \operatorname{sech} u\right) *\left(\frac{\delta}{u} n(u) \operatorname{sech} u\right)\right\} \\
n(u) & =Y\left(u^{2}-\delta^{2}\right) \frac{|u|}{\left(u^{2}-\delta^{2}\right)^{1 / 2}} \\
\delta(T) & =\frac{1}{2} \beta \Delta(T) .
\end{aligned}
$$

This corresponds to equation (2) of reference [5].
Here $Y(x)$ is the step function, $\Delta(T)$ is the energy gap according to BCS-theory, and $n(u)$ is the usual density of states of the electrons in a superconductor. The star denotes convolution products. For $E \rightarrow 0$ equation (6) reduces to

$$
\frac{1}{T_{1}^{\mathrm{S}}}=r K_{1} T \frac{2}{\mathrm{e}^{\beta \Delta(T)}+1} .
$$

For the numerical calculations we use for $\Delta(T)$ [10] :

$$
\begin{aligned}
& \frac{\Delta(T)}{\Delta(0)}=a(1-\theta)^{1 / 2}+(1-\theta) \times \\
& \quad \times\left[(1-a)+\left(1-\frac{a}{2}\right) \theta+\left(1-\frac{3 a}{8}\right) \theta^{2}\right] .
\end{aligned}
$$

In this equation $a=1.82, \theta=T / T_{\mathrm{c}}$, and $\Delta(0)=1.76 k T_{\mathrm{c}}$. If $\Delta=0\left(T \geqslant T_{\mathrm{c}}\right.$ or $\left.H \geqslant H_{\mathrm{c}_{2}}\right)$, then $F(u)$ in equation $(6)$ becomes equal to $F_{1}(u)$ and the value of $1 / T_{1}^{\mathrm{N}}$ as in the normal state is recovered.

It must be noted that $1 / T_{1}^{S}$ contains a discontinuity at a temperature determined by the equation $2 E=2 \Delta(T)$. This occurs only for T.L.S. with energy splitting $2 E$ such that $E<\Delta(0)$. In this case an enhancement of $1 / T_{1}^{\mathrm{S}}$ occurs, leading to a relaxation rate faster than in the normal metal at the same temperature. This additional relaxation is due to the fact that the T.L.S. might create or annihilate a pair of quasi-particles out of the BCS ground state. Such a mechanism of pair breaking is also responsible for the discontinuous behaviour of the ultrasonic absorption occurring in crystalline superconductors whenever $\hbar \omega>2 \Delta(T)$ [11]. The magnitude of the discontinuity can be calculated analytically :

$$
\Delta\left(\frac{1}{T_{1}^{\mathrm{s}}}\right)=r K_{1} T \frac{\pi}{2} \delta\left(1+\tanh ^{2} \delta\right) .
$$

Our calculations indicate that the discontinuity modifies the absorption and dispersion considerably at $T \lesssim T_{\mathrm{c}}$. The two quantities $K_{1}$ and $K_{3}$ were taken as free parameters in the numerical calculations. $C$ is a multiplicative factor which appears in the attenuation whatever the relaxation process might be. It determines the height of the plateau :

$$
l^{-1}=\frac{\pi}{2} C \frac{\omega}{v} \quad C=\frac{\mathcal{N} A \gamma^{2}}{2 \rho v^{2}} .
$$

From the magnitude of the plateau we get $C=5.5 \times 10^{-5}$. We calculate the relaxational attenuation taking into account only the direct process (phonons). The value of $K_{3}$ is adjusted such that we obtain the closest fit with the experimental results at the lowest temperatures where the absorption indeed exhibits a $T^{3}$-dependence. This $T^{3}$-dependence is characteristic for phonon-dominated relaxation and is also experimentally observed in amorphous insulators [7]. The corresponding curve is shown 
in figure 1 as a dashed line. The fit yields $K_{3}=3.5 \times 10^{9} \mathrm{~K}^{-3} \cdot \mathrm{s}^{-1}$. Adding to $T_{1}^{-1}$ the contribution of the electrons in the superconducting state we obtain the full line which agrees well with the attenuation as a function of temperature at the two frequencies used in the experiment [6] (Figs. 1 and 2). For $K_{1}$ we get $K_{1}=1.6 \times 10^{11} \mathrm{~K}^{-1} \cdot \mathrm{s}^{-1}$. In the

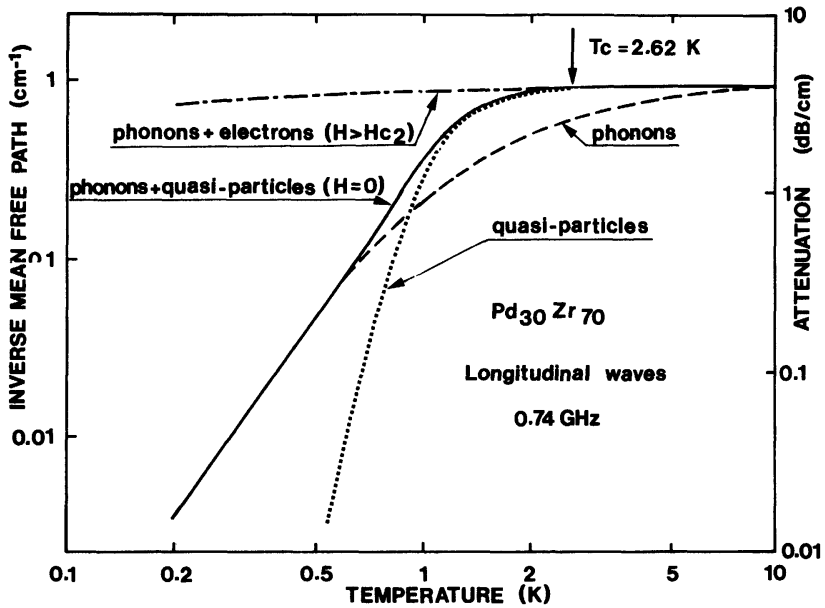

Fig. 1. - Calculated attenuation $l^{-1}$ originating from the relaxation of T.L.S. as a function of temperature. The calculation holds for longitudinal waves at a frequency $\omega / 2 \pi=0.74 \mathrm{GHz}$ for the superconducting amorphous alloy $\operatorname{Pd}_{30} \mathrm{Zr}_{70}$. The dashed line shows the contribution of phonons to the relaxation process whereas the dotted line represents the contribution of thermally excited quasiparticles only $\left(T<T_{\mathrm{c}}\right.$ and $\left.H=0\right)$. The dashed-dotted line shows the relaxation attenuation including the contributions of both phonons and electrons to the relaxation of the T.L.S. $\left(H>H_{\mathrm{c}_{2}}\right)$. The full line represents the attenuation taking into account the contributions of thermal phonons and quasi-particles to the relaxation rate of the T.L.S. $\left(T<T_{\mathrm{c}}\right.$ and $\left.H=0\right)$. There is no noticeable discontinuity of the slope of $l^{-1}$ at $T_{\mathrm{c}}$ on the scale used here. This holds even if one considers only the influence of electrons on the processes discussed here.

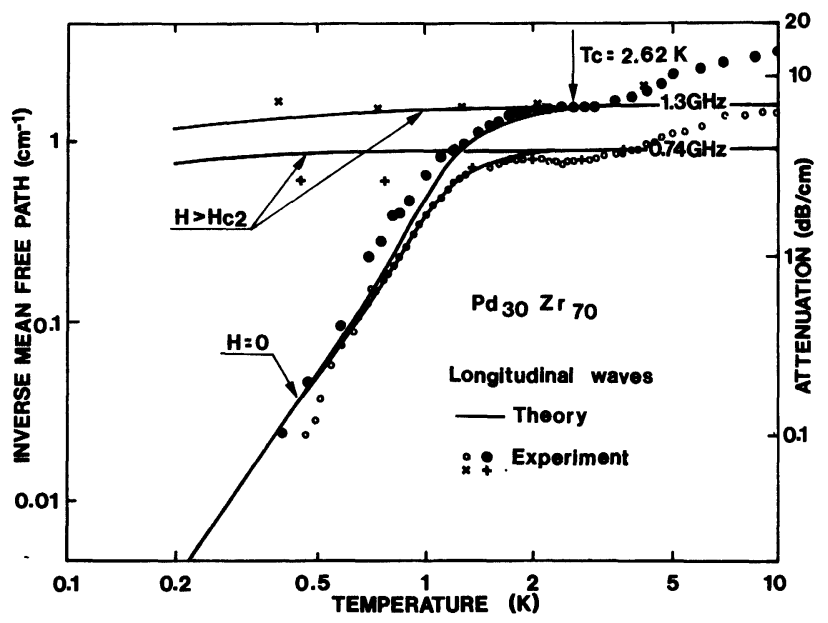

Fig. 2. - Measured ultrasonic attenuation as a function of temperature for longitudinal waves at the two frequencies $\omega / 2 \pi=0.74 \mathrm{GHz}$ and $1.3 \mathrm{GHz}$ (from [6]) together with theoretical curves. Circles represent experimental result without applied magnetic field, crosses with applied field parallel to the acoustical wave vector. The full lines show the calculated attenuation including the influence of phonons and electrons to $1 / T_{1} \quad(H=0$ and $\left.H \gtrsim H_{\mathrm{c}_{2}}\right)$. As can be seen there is very good agreement between theory and experiment. The increase of the measured attenuation at temperatures above $3 \mathrm{~K}$ is not discussed here. case of an applied magnetic field $H \gtrsim H_{\mathrm{c}_{2}}$ only electrons in the normal state and the thermal phonons contribute to $T_{1}^{-1}$. The calculated absorption is shown in figure 1 by the dashed-dotted line. The comparison with the experiments is shown in figure 2 . For completeness we have calculated the attenuation taking into account only the relaxation of T.L.S. by thermally excited quasi-particles. It is represented by the dotted line in figure 1 . We have also checked that the influence of higher-order phonon processes is negligible unless coupling constants are used which are unreasonably high.

3. Discussion. - The magnitudes of $K_{1}, K_{3}$ and $C$ obtained from the numerical calculation allow one to deduce some physical quantities relevant to the T.L.S. in $\mathrm{Pd}_{30} \mathrm{Zr}_{70}$. From $K_{1}$ we get $\tilde{\rho} K_{\mathrm{e}}=0.9$ which is higher than found in other amorphous non-superconducting metals : 0.3 [12] and 0.16 [4] for $\mathrm{PdSiCu}$ and 0.25 for $\mathrm{NiP}$ [13], respectively. From $C$ we get straightforwardly

$$
\mathcal{N} A \gamma_{\mathrm{L}}^{2} / 2=8 \times 10^{7} \mathrm{erg} \cdot \mathrm{cm}^{-3}
$$

$\left(\rho=7.8 \mathrm{~g} / \mathrm{cm}^{3}, v_{\mathrm{L}}=4.4 \times 10^{5} \mathrm{~cm} / \mathrm{s}\right)$ in reasonable agreement with the value deduced from sound velocity measurements [14] $\left({ }^{2}\right)$. One way to obtain full agreement is to modify the distribution function $P(E, r)$ as has been done in the case of $\beta$-alumina [8]. $K_{3}$ is proportional to $\gamma_{\mathrm{L}}^{2} / v_{\mathrm{L}}^{5}+2 \gamma_{\mathrm{T}}^{2} / v_{\mathrm{T}}^{5} \cdot \gamma_{\mathrm{T}}, \gamma_{\mathrm{L}}$ and $v_{\mathrm{T}}$ are not known for $\operatorname{Pd}_{30} \mathrm{Zr}_{70}$. By comparison with other amorphous metals we assume $v_{\mathrm{L}} \simeq 2.2 v_{\mathrm{T}}$. Quite accurately we can therefore say that only transverse phonons are effective in relaxation rates. With $v_{\mathrm{T}}=2 \times 10^{5} \mathrm{~cm} / \mathrm{s}$ and neglecting the contribution of longitudinal phonons to $K_{3}$, we find $\gamma_{\mathrm{T}}=0.8 \mathrm{eV}$ which indicates a rather strong coupling between T.L.S. and transverse phonons. Using $\mathcal{N} A / 2=2 \times 10^{33} \mathrm{erg}^{-1} \cdot \mathrm{cm}^{-3}$ obtained from the specific heat measurements [15] we deduce $\gamma_{\mathrm{L}}=0.13 \mathrm{eV}$ as a lower limit. $\gamma_{\mathrm{L}}$ is smaller than $\gamma_{\mathrm{T}}$ and the above approximation is therefore justified.

In their numerical calculations, Black and Fulde have found an abrupt change of the attenuation slope at $T_{\mathrm{c}}$ (see figure 2 of [5]). We do not find such a behaviour. We attribute this mainly to the larger value of $\tilde{\rho} K_{\mathrm{e}}=0.9$ as compared to 0.2 in [5]. Of course the additional contribution of the thermal phonons to the relaxation rate of the T.L.S. further suppresses any possible discontinuity at $T_{\mathrm{c}}$. Finally in our opinion the value of $r_{m}$ used in the numerical calculations also influences the occurrence of such an abrupt change. $r_{m}$ reflects those T.L.S. in the absorption having a slow relaxation rate and hence determines the extent of the plateau. We used values for $r_{m}$ from

(2) The sound velocity measurements yield

$$
\mathcal{N} A \gamma_{\mathrm{L}}^{2} / 2 \simeq 3.6 \times 10^{7} \mathrm{erg} / \mathrm{cm}^{3}
$$

and not $\mathcal{N} A \gamma_{\mathrm{L}}^{2} / 2=1.4 \times 10^{7} \mathrm{erg} / \mathrm{cm}^{3}$ as reported in [14] (Fig. 1A). Apparently the factor $\ln 10$ has been omitted when calculating $\mathcal{N} A \gamma_{\mathrm{L}}^{2} / 2$ in [14]. 
$10^{-3}$ to $10^{-10}$. For $r_{\mathrm{m}} \leqslant 10^{-4}$ the experimental attenuation curve is properly reproduced in the temperature range of interest here (see also Appendix).

The experimental results in $\mathrm{Pd}_{30} \mathrm{Zr}_{70}$ as shown in figure 2 exhibit (for $T>3 \mathrm{~K}$ ) an excess attenuation in addition to the plateau. We do not discuss this excess attenuation here. It might be similar to a relaxational mechanism commonly observed in amorphous insulators and in other amorphous metals $[16,17]$.

We have also calculated the relative variation of the velocity of sound as a function of temperature (equation (3)) using the parameters deduced from the fit of the attenuation (equation (1)). We added the contribution due to resonant interaction of the T.L.S. with phonons which varies as $C^{\prime} \ln \left(T / T_{0}\right) . T_{0}$ is a reference and $C^{\prime}$ has to be taken from experiment [14]. The result is shown in figure 3. As can be seen, there is a discontinuous slope of the relative variation of the sound velocity at $T_{\mathrm{c}}$. This can be simply explained. The inner integrals in equations (1) and (3) can be solved analytically. Provided $\omega T_{1}^{\mathrm{m}} \ll 1$, in equation (1) this integral results in $\pi / 2$ whereas in equation (3) in $\ln \left(\omega T_{1}^{\mathrm{m}}(E, T)\right)$ [8]. Therefore all details of the relaxational processes have vanished from the calculation

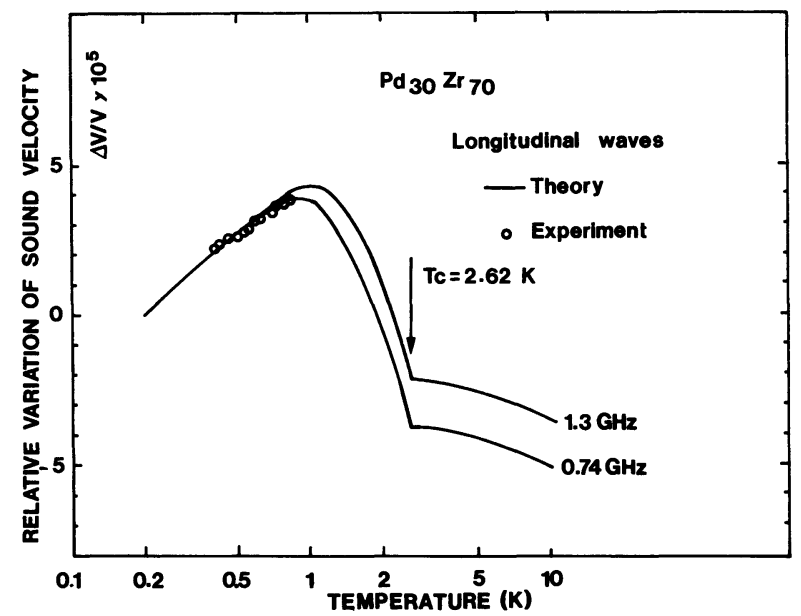

Fig. 3. - Calculated relative velocity change $\Delta v / v$ as a function of temperature for longitudinal waves at two frequencies $\omega / 2 \pi=0.74 \mathrm{GHz}$ and $\omega / 2 \pi=1.3 \mathrm{GHz}$. The calculations are carried out using $K_{1}$ and $K_{3}$ obtained from the fit of the attenuation (Fig. 2). The resonant contribution to $\Delta v / v$ varying logarithmically with temperature is added (from [14], open circles). The corresponding coefficient is $C^{\prime}=3.0 \times 10^{-5}$. In contrast to the behaviour of the attenuation, the velocity shift should exhibit a change in slope at $T_{\mathrm{c}}$. No detailed experimental measurements of $\Delta v / v$ around $T_{\mathrm{c}}$ are yet available. of the attenuation. On the other hand the relaxation rate is still explicitly present in the velocity through $T_{1}^{\mathrm{m}}$ which of course contains the rapid change of $T_{1}^{-1}$ caused by the electrons at $T_{\mathrm{c}}$. The change of slope depends on the relative weight of the phonon and electron processes and hence on the ratio $K_{3} / K_{1}$. At present only preliminary measurements of $\Delta v(T) / v$ at $T \simeq T_{\mathrm{c}}$ have been carried out [14] and therefore a detailed comparison between theory and experiment is not possible.

Furthermore, we expect that the acoustic anomalies observed in polycrystalline $\mathrm{NbZr}$ containing tunnelling centres [18] can be explained in an analogous way.

4. Conclusion. - In summary, we should like to emphasize that within the framework of the tunnelling model, the simultaneous coupling of the tunnelling states to thermal phonons and to electrons satisfactorily explains the acoustic absorption in $\mathrm{PdZr}$ at very low temperatures. Our calculations indicate that transverse waves couple more strongly to T.L.S. than longitudinal ones. In contrast to the absorption, the slope of the velocity shift as a function of temperature should exhibit a pronounced change at $T_{\mathrm{c}}$. Therefore, accurate and comprehensive measurements of $\Delta v(T) / v$ are highly desirable. They should provide a further test of the physical ideas presented in this paper.

Acknowledgments. - One of us (W.A.) is grateful to the Fraunhofer-Institut at Saarbruecken for the generous support of this work by allowing the use of its computer facilities. He also thanks $H$. Willems for his hints concerning the numerical calculations.

Appendix. - The calculation of the relaxational attenuation $l^{-1}(T)$ and $\Delta v(T) / v$ needs a knowledge of the relaxation rate $1 / T_{1}$. The processes which contribute to the relaxation of T.L.S. can all be described by analytical expressions except for the relaxation due to thermally excited quasi-particles. This contribution can be obtained only through a numerical calculation. We shortly explain how this is done and how the numerical integration of equations (1) and (3) was carried out. The quantity which is of interest in this context is

$$
1 / T_{1}^{\mathrm{S}}=r K_{1} T F(\beta E) .
$$

Here we use for $F(X)$ a new form which is equivalent to the expression giving $F(u)$ in equations (6).

$$
\begin{aligned}
F(X)=\frac{1}{4(X+d)} \tanh X \cdot\left\{\int_{-1}^{+1} \frac{\mathrm{d} x}{\sqrt{1-x^{2}}} \frac{\frac{(X+d)^{2}}{x^{2}}-\left(X^{2}+d^{2}\right)}{\sqrt{1-\left(\frac{X-d}{X+d}\right)^{2} x^{2}}}\left(\tanh P_{\mathrm{e}}+\tanh Q_{\mathrm{e}}\right)+\right. \\
\left.+Y\left(X^{2}-d^{2}\right) \int_{-1}^{+1} \frac{d}{\sqrt{1-x^{2}}} \frac{\left(X^{2}+d^{2}\right)-(X-d)^{2} x^{2}}{\sqrt{1-\left(\frac{X-d}{X+d}\right)^{2}}}\left(\tanh P_{\mathrm{i}}+\tanh Q_{\mathrm{i}}\right)\right\}
\end{aligned}
$$


where

$$
\begin{array}{rlrl}
X & =\beta E & d & =\beta \Delta \\
P_{\mathrm{e}} & =\frac{1}{2}\left(X+\frac{X+d}{x}\right) & P_{\mathrm{i}} & =\frac{1}{2}(X+(X-d) x) \\
Q_{\mathrm{e}} & =\frac{1}{2}\left(X-\frac{X-d}{x}\right) & Q_{\mathrm{i}} & =\frac{1}{2}(X-(X-d) x) .
\end{array}
$$

The second term gives no contribution when $X<d$ (or $E<\Delta(T)$ ). Indeed these two integrals are of the form

$$
I=\int_{-1}^{+1} \frac{\mathrm{d} x}{\sqrt{1-x^{2}}} f(x)
$$

which may be calculated by a single-step numerical integration with the Gauss-Tchebichev method which uses :

$$
I=\frac{\pi}{n} \sum_{i=1}^{n} f\left(x_{i}\right)
$$

where

$$
x_{i}=\cos \left(\frac{2 i-1}{2 n} \pi\right)
$$

denotes the $n$ roots of the Tchebichev polynomial of degree $n$. We have used $n=10$. The integrand $\left(1-x^{2}\right)^{-1 / 2} f(x)$ shows singularities at the two limits

of the integration domain. The method used here eliminates the difficulties occurring at the singular points at $x= \pm 1$. It can also be applied to other numerical problems in superconductivity where the density of states of the electrons enters.

In addition we used the exact value of the jump in $1 / T_{1}^{\mathrm{S}}$ occurring when $2 \Delta(T)=2 E$ (Eq. (8)), in order to check the accuracy of the numerical calculations.

The double integral necessary to obtain both $l^{-1}$ and $\Delta v / v$ is calculated in two steps. The inner integral is evaluated according to the trapezoidal rule with a variable interval fitted to the variation of the function. If the lower bound of the integration $r_{\mathrm{m}}$ is smaller than the value of $\omega T_{1}$ at higher temperatures and energy studied, the value of the integral becomes independent of $r_{\mathrm{m}}$. The outer integral is calculated with a standard method with constant interval. The lower integration limit is (in temperature units) $E_{\mathrm{m}}=0.01 T$. Because of the $\operatorname{sech}^{2}(\beta E)$ function in equations (1) and (3) it is sufficient to take $E_{\mathrm{M}}=4 T$ for the upper bound of the integral.

\section{References}

[1] BLACK, J. L., in Metallic Glasses, edited by H. J. Güntherodt (Springer-Verlag, N. Y.) 1980.

[2] Bellessa, G., Doussineau, P. and Levelut, A., J. Physique Lett. 38 (1977) L-65.

[3] Doussineau, P., Legros, P., Levelut, A. and Robin, A., J. Physique Lett. 39 (1978) L-265.

[4] Golding, B., Graebner, J. E., Kane, A. B. and Black, J. L., Phys. Rev. Lett. 41 (1978) 1487.

[5] Black, J. L. and Fulde, P., Phys. Rev. Lett. 43 (1979) 453.

[6] Weiss, G., Arnold, W., Dransfeld, K. and Güntherodt, H. J., Solid State Commun. 33 (1980) 111 ;

Weiss, G., Arnold, W. and Güntherodt, H. J., J. Physique Colloq. 41 (1980) C 8-742.

[7] JäckıE, J., Z. Phys. 257 (1972) 212.

[8] Doussineau, P., Frenois, Ch., Leisure, R. G., Levelut, A. and Prieur, J.-Y., J. Physique 41 (1980) 1193.

[9] Phillips, W. A., J. Low Temp. Phys. 7 (1972) 351.

[10] This formula was chosen because it is simple and reproduces $\Delta(T)$ in very good agreement with more sophisticated equation (see Bliss, E. S. and Rayne, J. A., Phys. Rev. 177 (1969) 673).

[11] Helme, B. G. M., Hilton, P. A., Meredith, D. J. and WigMORE, J. K., J. Phys. F : Metal Phys. 7 (1977) 427, and references therein.

[12] Doussineau, P., J. Physique Lett. 42 (1981) L-83.

[13] Doussineau, P. and Robin, A., in Phonon scattering in solids, edited by H. H. Maris (Plenum Press, N. Y.) 1980, p. 65.

[14] WeIss, G. and ARNOLD, W., in Liquid and amorphous metals, edited by E. Luescher and H. Coufal (Sijthoof u. Noordhoft) 1980, p. 657.

[15] Graebner, J. E., Golding, B., Schutz, R. J., Hsu, F. S. L. and CHEN, H. S., Phys. Rev. Lett. 39 (1977) 1480.

[16] Dutort, M., Phys. Lett. A 50 (1974) 221.

[17] See for example : Hunklinger, S. and ARnold, W., in Physical Acoustics, Vol. XII, edited by W. P. Mason and R. N. Thurston (Academic Press) 1976, p. 155.

[18] Thomas, N., Arnold, W., Weiss, G. and Loehneysen, H. V., Solid State Commun. 33 (1980) 523. 